\title{
A SPLINE-BASED ADAPTIVE FILTER FOR THE REMOVAL OF BLOCKING ARTIFACTS IN IMAGE SEQUENCES CODED AT VERY LOW BITRATE
}

\author{
Roberto Castagno
}

Signal Processing Laboratory

Swiss Federal Institute of Technology

CH-1015 Lausanne, Switzerland

email: castagno@lts.de.epfl.ch
Juan Andrei Villaroel

\author{
Swiss Federal Institute of Technology \\ CH-1015 Lausanne, Switzerland \\ email:villar@desun1.epfl.ch
}

\begin{abstract}
In this paper, a method for the reduction of blocking effect in image sequences coded at very low bitrate is presented.

The proposed operator is capable of adapting its behaviour to the local characteristics of the images. In areas containing details, the degradation introduced by block-based coding is often masked by the texture itself and a satisfactory visual effect can be achieved by simply leaving the area unchanged.

In uniform areas, instead, the coarse quantization normally used at very low bitrate- results in visible steps in the gray level. A local action along the border is not sufficient for the removal of the artifact (specially when the frames are later viewed in a sequence). In this case, a spline interpolation is performed on the gray levels of pixels located on a wider area across the border. The extension of the filtered zone inside the blocks is evaluated by means of an index of activity so as to maximize the smoothing effect, while preserving the details inside the blocks.
\end{abstract}

\section{INTRODUCTION}

Blocking effect is one of the most typical -and at the same time most annoying- artifacts that affect images that have been coded by means of block based techniques. Pixels belonging to adjacent blocks are treated without their mutual correlation being taken into account, which results in quite visible block boundaries.

The reduction of this artifact can result in a significant improvement of the overall visual quality of the image. Various examples can be found in the literature in which this problem is tackled with different approaches: some authors suggest to appropriately select the blocks during the coding phase itself (typically by choosing overlapping blocks), others propose post-processing techniques which consist in filtering the image after the decoding.

The characteristics of the human visual system also play an important role in the perception of blocking related degradations, and methods for the reduction of these artifacts try to match the sensitivity of the human eye. For the above mentioned reasons, non-linear and space-variant techniques prove quite attractive for the solution of this problem [1].

In this paper, we propose a simple but effective post-processing method for the reduction of blocking effect in sequences coded at very low bitrate. The sensitivity of the human eye is taken into account by performing the post-processing only in areas where the artifact is not masked by the presence of details. The actual filtering is performed by means of third order spline functions: the range of action of the filter in the neighborhood of the border is determined by means of a nonlinear estimator, which detects the presence, inside the blocks, of details which might be damaged by the interpolation.

\section{ADDRESSED PROBLEM}

The proposed method has been developed in order to improve the performance at very low bitrate of the coding scheme presented in $[2,3]$ within the framework of MPEG4 standardization process. In this scheme, frames are segmented according to a variable depth quadtree partitioning process, and the coding strategy for each of the blocks is chosen within a repertoire of intra-frame and inter-frame techniques; among the former are fractal coding, DCT, bi-level, and the latter include motion-compensated techniques. The depth of the partition (and, consequently, the size of the blocks) and the coding technique for each block are chosen so as to optimize the tradeoff between bitrate and distortion. The above mentioned optimization process requ- 
ires the blocks to be statistically independent. This constraint excludes the possibility of using overlapping blocks. Therefore post-processing appeared to be the most attractive approach to take, also considering that the structure of the quadtree partition -although rather complex-is sent along in the bitstream and is therefore known at the decoder side.

Very low bitrate coding typically produces different kinds of artifacts in images. Highly detailed areas are strongly degraded and it is difficult to exploit the surviving details for enhancement. Often "no filtering is the best filtering" when block boundaries cross detailed zones, also because the blocking effect is well masked by the detail themselves. More uniform areas are instead further smoothed by the coding; in addition to that, coarse quantization often produces a "patchwork" effect: a smooth change of luminance across a block border can result in a step in the decoded image, if neighboring samples fall into different quantization intervals. The visual effect is very annoying, both because the human vision is very sensitive to edges in uniform areas and because -due to the so called Mach effect [4]- it perceives a higher step in luminance than the actual one in the gray level. When images are later viewed in a sequence, the patches vary in position and size, which further worsens the visual quality.

A local action along the borders in uniform areas (e.g. a linear smoothing of a strip of pixels across the borders) reduces the visual impact of the luminance step and contrasts the Mach effect. Such approaches are efficient if details are sufficiently preserved and can be exploited for the filtering. In [5] local slopes are evaluated and modified in order to achieve global smoothness, in [6] the proposed rational filter performs directional filtering according to the local characteristics of the texture. However, such methods do not solve the problem of the luminance differences inside the blocks due to the quantization error and loose efficiency in case of badly preserved details, which are the most common problems at very low bitrate.

On the other hand, a global smoothing has two major drawbacks: the first is the risk of further damaging details inside the blocks, the second is the excessive homogeneity (sometimes referred to as "cartoon effect") which results from the smoothing of large areas of the image.

The proposed solution, described in detail in the next sections, is a compromise between the above mentioned approaches, and consists in extending the filtering action from the border towards the interior of the blocks until a detail is found or a maximum distance from the border is reached.

\section{THE PROPOSED METHOD}

\subsection{Selection of appropriate action}

As mentioned in the introduction, the algorithm assumes knowledge of the position of the block boundaries; this is straightforward when the coding scheme is based on a fixed block partition (e.g. H.263 or MPEG2). In the case of the dynamic coding scheme[3], information about the structure of the quadtree is sent along in the bitstream and is therefore available to the decoder.

The post-processing takes place along the block boundaries in the same order as the successive splittings of the image block, starting from the full frame. The first step of the filtering procedure consists in the selection og the most appropriate form of action according to the local characteristics of the image region crossed by the block boundary.

At very low bitrate, the preservation of details is severely impaired, thus making it very difficult to envisage techniques that could exploit the presence of details for some kind of directional filtering. On the other hand, plain linear smoothing in textured areas would blur the details, resulting in further degradation of the image. Furthermore, it should be noted that the very presence of details masks quite well the block boundaries: "no filtering" seems to be the most appropriate technique in textured areas at very low bitrate.

In order to evaluate which border areas should be altered by filtering, two $3 \times 3$ windows are taken into account on the two sides of the border. In Fig. 1 we show the situation when pixels on row $n$ are filtered along a vertical border. The two windows are shown in dotted lines: let us refer to the left and the right one as $W i n_{l}$ and $W i n_{r}$ respectively.

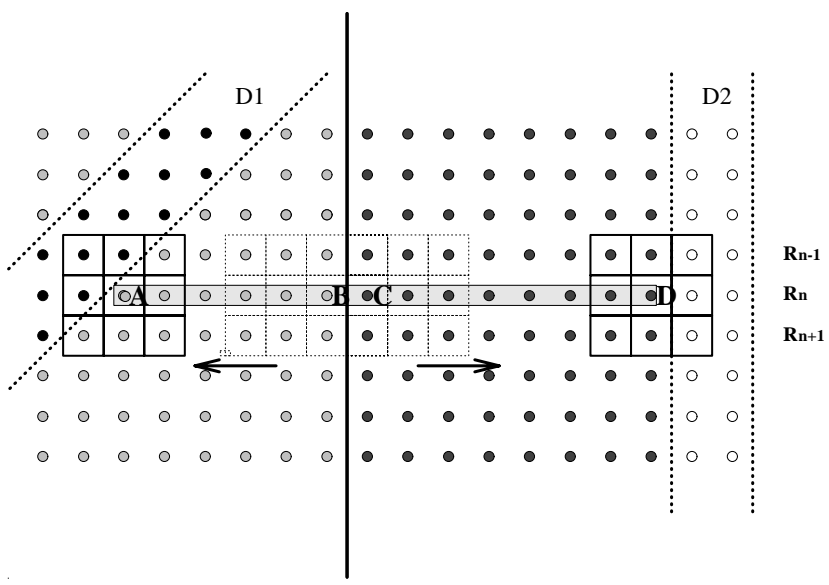

Figure 1: A simple example of how the proposed methods behaves in the presence of detail inside the blocks 
The gray level means $\mu_{l}$ and $\mu_{r}$ and variances $\sigma_{l}$ and $\sigma_{r}$ are evaluated over windows $W_{i n}$ and $W_{i n}$ respectively. Filtering takes place only if the following conditions are met:

$$
\begin{aligned}
\sigma_{l} & <\sigma_{t h 1} \\
\sigma_{r} & <\sigma_{t h 1} \\
\left|\mu_{l}-\mu_{r}\right| & <\mu_{t h}
\end{aligned}
$$

Equations 1 and 2 assure that the two sides of the border are not textured (i.e. the gray level variances are beolw a given threshold $\left.\sigma_{t h 1}\right)$. Equation 3 prevents from the filtering taking place in case $W i n_{l}$ and $W i n_{r}$ are uniform, but the difference of average gray level is high and due to a real step in the image which should be preserved, rather than to the blocking effect.

If the three conditions above are met, the filtering action takes place as described in the following section.

\subsection{Filtering}

Let us refer ourselves again to Fig. 1 and suppose the three conditions $(1-3)$ are met. The two $3 \times 3$ windows are moved -one pixel at a time- away from the border into the two adjacent blocks, and at each step the variance is evaluated again. Each window stops when it detects the presence of a detail, i.e. when the variance becomes higher then a threshold $\sigma_{t h 2}$. In the depicted example, the window on the left stopped when it met the darker pixels of detail D1, the window on the right side stopped when it detected the white pixels of detail D2.

A three-point 3rd order spline is then evaluated in order to modify the gray level of pixels between the final locations of the two windows: the fixed points are the central pixels of the windows (in Figure 1, points A and $D$ respectively), and the average gray level of the two pixels on the two edges of the border (i.e. $(B+C) / 2)$. The pixels which are affected by the interpolation are those surrounded by a shaded rectangle. The same operation is then performed on the following row $R_{n+1}$ and so on, along the whole border.

In this way, the operator performs a filtering robust enough to significantly reduce the "patchwork" effect, but sensitive to the presence of details inside the block, which are preserved.

It is possible to keep a memory of the characteristics of the image area spanned by the two sliding windows, by comparing the threshold $\sigma_{t h 2}$ with the summation of the variances encountered in all the intermediate positions, rather than with the single variance at each position. In this way, if very tiny details are present inside the block, the depth of the filtering inside the two blocks will be reduced, as well as the risk of unwillingly smoothing large areas of fine texture.

In order to better control the action of the filter, it is also possible to limit a priori the width of the filtered area: in the simulations we set one eighth of the block size as a limit.

\section{SIMULATION RESULTS}

As it is often the case, it is difficult to judge by objective parameters the performance of the method. The blocking effect is so concentrated that it affects only marginally the Mean Square Error (MSE), which, moreover, is already quite high at very low bitrate. Subjective evaluation, especially when sequence processing is involved, remains the most valuable one.

In Fig. 2(a) the original frame 0 of sequence is shown; the same frame after the coding-decoding loop at 10 $\mathrm{Kb} / \mathrm{s}$ is shown in Fig. 2(b). The blocking effect is clearly visible, as well as the loss of details (e.g. on the ship) and the overall smoothing (e.g. sea).

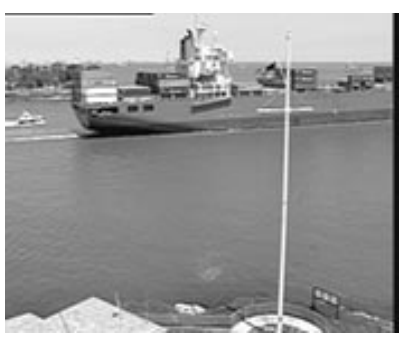

(a)

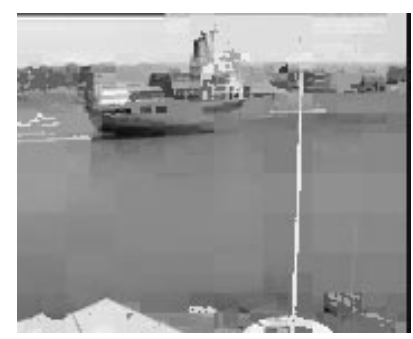

(b)
Figure 2: (a) Frame 0 of sequence "Container ship". (b) The same frame after being compressed at $10 \mathrm{~kb} / \mathrm{s}$.

Figures 3 (a) and 3(b) demonstrate how the filter performs the reduction of the blocking artifacts in Fig. 2(a).

In particular, Fig.3(a) shows how the variance detector selects the areas of intervention. The borders between blocks are depicted: black pixels correspond to border areas where high variance has been detected along the border (i.e. above threshold $\sigma_{t h 1}$ in Equations 1 and 2), and no filtering has therefore taken place. A remarkable example of the performance of the detector can be seen on the diagonal step at the bottom of the image: in areas where the block borders intersect the step, the variance has been correctly detected, no filtering is performed, and the step is not blurred. Border areas where filtering has instead taken place have been colored in gray. The extreme points of the splines used in the filtering have been plotted in white: it can be clearly seen that the filtering affected only the uniform areas, but correctly stopped before 


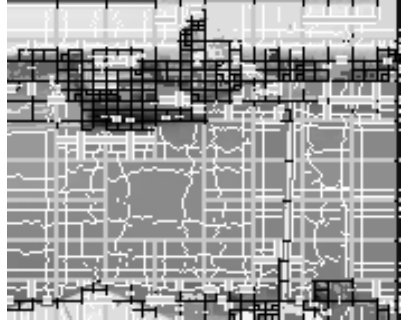

(a)

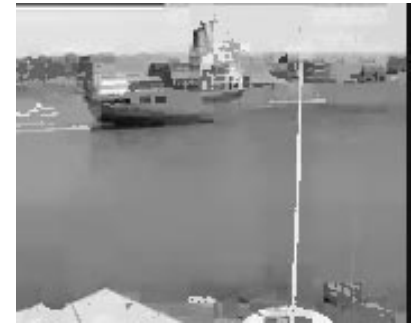

(b)
Figure 3: (a) Analysis of the filtering action of the proposed algorithm. In this test frame, the quadtree partition structure is superposed to the image, so as to show the zones where the filtering took place. Black pixels represent zones where detail was found and no filtering was performed. Gray pixels indicate borders in uniform areas, which were affected by the filtering. White pixels indicate the extreme points of the splines used for the interpolation.(b) The same frame of Fig.2(b) after post-processing.

damaging any detail inside the blocks, e.g. the flag pole or the tiny waves behind the small boat, left on the top.

The overall result of the post-processing can be seen in Figure 3(b): the blocking effect has been strongly reduced in uniform areas, still avoiding an excessive smoothing (for example the shadow of the ship on the sea is preserved). Other zones have been left unchanged, and details (at least what was left after very low bitrate coding) are preserved.

Figures 4(a) and 4(b) give an idea of the effect of the filtering procedure on the luminance values.
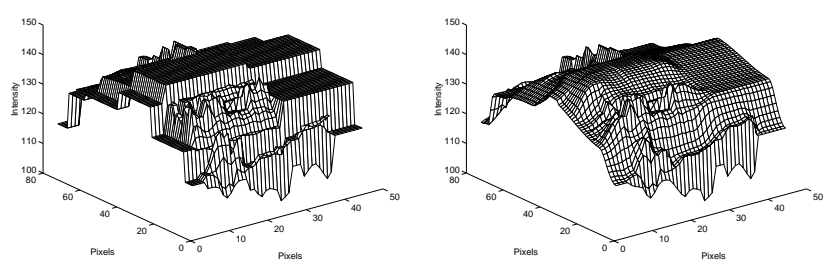

(a)

(b)

Figure 4: A 3-D representation of the luminance levels of a $80 \times 50$ area of frame 0 of sequence Container Ship. (a) Blocking effect is visible. (b) Blocking boundaries have been smoothed away by post-processing, but details in foreground are preserved.

Gray levels of a $80 \times 50$ window of the image in Figures 2(b) and 3(b) are plotted on the $z$ axes in Fig. 4(a) and 4(b) respectively. It can be seen that the luminance steps due to the blocking effect are properly smoothed away, but the details in the foreground are well preserved, despite their relatively small amplitude.

\section{CONCLUSIONS}

In this paper, a simple method for the reduction of blocking artifacts in image sequences coded at very low bitrate has been presented.

The method copes with the twofold characteristic of the artifact. In textured areas action has to be limited, in order to preserve the few remaining details from further blurring. In uniform areas the filtering must penetrate towards the interior of blocks, in order to perform a robust smoothing, yet avoiding to damage details located inside the blocks.

Decision about the action to take and the limits of the filtering is made based on the level of activity measured by means of a variance detector.

\section{REFERENCES}

[1] B. Ramamurthi and A. Gersho. "Nonlinear SpaceVariant Postprocessing of Block Coded images". IEEE Transactions on Acoustics, Speech, and Signal Processing, Vol. 34, No. 5, pp. 1258-1267, October 1986.

[2] T. Ebrahimi, F. Bossen, R. Castagno, C. De Sola, C. Le Buhan, L. Piron, E. Reusens, and V. Vaerman. "Dynamic Coding of Visual Information". MPEG4 proposal JTC/sC29/WG11/M0320, Signal Processing Laboratory, Swiss Federal Institute of Technology, 1995.

[3] E. Reusens, R. Castagno, C. Le Buhan, L. Piron, and M. Kunt. "Dynamic Video Coding - A Review -". In Proceedings of the International Conference on Image Processing ICIP, Lausanne, Switzerland, September 1996.

[4] A. K. Jain. Fundamental of Digital Image Processing. Prentice Hall, 1989.

[5] H. Paek, J.W. Park, and S.-U. Lee. "Non-Iterative Post-Processing Technique For Transform Coded Image Sequence". In Proceedings of the International Conference on Image Processing ICIP, Vol. 3, pp. 208-211, Washington, USA, October 1995.

[6] R. Castagno and G. Ramponi. "A Rational Filter for the Removal of Blocking Artifacts in Image Sequences Coded at Low Bitrate". In Proceedings of the VIII European Signal Processing Conference EUSIPCO, Trieste, Italy, September 1996. 